\title{
Antipsychotikum zur Augmentierung?
}

Fragestellung: Wie wirksam ist Ziprasidon als Augmentierungstherapie bei depressiven Patienten, die erfolglos mit Escitalopram behandelt wurden?

Hintergrund: Antipsychotika wie Quetiapin, Aripiprazol und Olanzapin werden zunehmend auch zur Augmentierungstherapie bei depressiven Patienten eingesetzt, die erfolglos mit einem Antidepressivum behandelt wurden. Quetiapin ist in dieser Indikation bei Therapieresistenz zugelassen, in den USA auch Olanzapin und Aripiprazol. Die Studie ging der Frage nach, ob auch für Ziprasidon ein augmentierender Effekt einer Antidepressivabehandlung nachgewiesen werden kann.

Patienten und Methodik: Zwischen 2008 und 2013 wurden an drei Zentren in den USA 311 ambulante Patienten mit unipolarer, nicht psychotischer Depression (HAMD-Mittelwert 20,0 zu Beginn) zunächst acht Wo-

Papakostas GI, Fava M, Baer L et al. Ziprasidone augmentation of escitalopram for major depressive disorder: Efficacy results from a randomized, double blind, placebo-controlled study. Am J Psychiatry. 2015; 172: $1251-8$ chen offen mit Escitalopram (mittlere Dosis zirka $20 \mathrm{mg}$ ) behandelt. Im Durchschnitt hatten die Patienten auf einen vorherigen Therapieversuch mit einem ausreichend dosierten Antidepressivum über mindestens sechs Wo- chen nicht angesprochen. Nach Ablauf der offenen Phase wurden alle 139 Patienten, die eine ungenügende Therapieresponse auf das Antidepressivum zeigten (HAMD-Mittelwert zirka 17,5), auf zwei Therapiearme randomisiert: 71 Patienten erhielten für weitere acht Wochen zusätzlich Ziprasidon (mittlere Dosis zirka $98 \mathrm{mg}$ ) und 68 Patienten zusätzlich Placebo. Primärer Outcome war Response nach acht Wochen (HAMD-17 > 50\% Besserung). Sekundäre Endpunkte waren die Hamilton-AngstSkala und eine visuelle Analogskala für Schmerzsymptome.

Ergebnisse: Die Kombination mit Ziprasidon war im primären Outcome gegenüber der Placeboaugmentation überlegen: Die Responserate lag bei 35,2 versus 20,5\%. Auch zeigte sich eine Überlegenheit bei der Reduktion depressiver Symptome: Rückgang um 6,4 (SD 6,4) versus 3,3 (SD 6,2) Punkte auf der HAMDSkala. Im wichtigsten sekundären Outcome, dem HAMD-AngstWert, war die Kombination ebenfalls überlegen, nicht aber in der visuellen Analogskala für Schmerzen. Etwa einer von drei Patienten brach die Kombinationsbehandlung vorzeitig ab.

Schlussfolgerungen: Die Autoren folgern, dass eine Kombination von Escitalopram mit Ziprasidon einer Weiterbehandlung mit Escitalopram allein bei depressiven Patienten überlegen ist, die sich auf acht Wochen Escitalopram nicht ausreichend gebessert haben.

\section{- Kommentar von Klaus Lieb, Mainz}

\section{Antipsychotika bei Depression zurückhaltend einsetzen}

Aufgrund der chemischen Eigenschaft mit einer 5- $\mathrm{HT}_{2 \mathrm{a}}$-Blockade und partiell $5-\mathrm{HT}_{1 \mathrm{a}}$-agonistischen Eigenschaft sowie der Hemmung der Serotonin- und Noradrenalin-Wiederaufnahme, die auf dem Niveau von Duloxetin und Venlafaxin liegen soll, qualifiziert sich Ziprasidon grundsätzlich als antidepressive Augmentierungsstrategie. Die qualitativ hochwertige Studie liefert dafür auch einen ersten Hinweis. Wenn weitere Studien diesen Effekt bestätigen sollten, wäre Ziprasidon als Augmentierungsstrategie bei therapieresistenter Depression durchaus in Erwägung zu ziehen, was gegenüber Quetiapin und Olanzapin den Vorteil der geringeren metabolischen Komplikationsrate hätte. Dennoch ist aus mehreren Gründen Zurückhaltung geboten: 1) In der Studie hatten die Patienten in der gegenwärtigen Erkrankungsphase im Durchschnitt nur auf ein vorheriges Antidepressivum nicht angesprochen. In einem solchen Fall hätte man die Umstellung auf ein anderes Antidepressivum, eine Kombinationstherapie, die Kombination mit Psychotherapie oder eventuell mit Lithium bevorzugt. 2) Ziprasidon gehört unter den Zweitgenerationsantipsychotika zu den Substanzen mit dem höchsten Risiko für QT-Zeit-Verlängerungen, was gerade in der Kombination mit Ecitalopram, das selbst die QT-Zeit verlängert, problematisch ist. 3) Die HAMD-Wertunterschiede nach acht Wochen Kombination versus Placebo sind mit etwa drei Punkten sehr klein, und der Placeboeffekt war zusätzlich sehr klein - insofern ist fraglich, ob die Ergebnisse klinisch relevant sind (Effektstärkenunterschiede lassen sich wegen fehlender Angaben von Absolutwerten nicht ausrechnen). 4) Etwa einer von drei Patienten brach wegen Nebenwirkungen die Kombinationstherapie ab. Auch kam es recht häufig zu Müdigkeit, was schon in Schizophreniestudien beschrieben wurde, obwohl Ziprasidon keine relevante Affinität zu histaminergen oder cholinergen Rezeptoren hat. 5) Studienergebnisse an rund zweimal 70 Patienten sind oft Zufallsbefunde. Insofern müssen unbedingt weitere Studien folgen, bevor man Ziprasidon in dieser Indikation in Erwägung zieht.

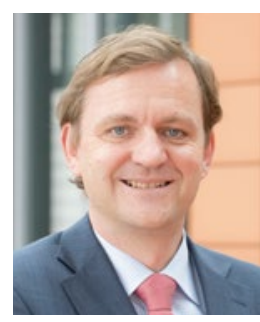

Prof. Dr. med. Klaus Lieb, Mainz

Direktor der Klinik für Psychiatrie und Psychotherapie, Universitätsmedizin Mainz E-Mail: klaus.lieb@unimedizin-mainz.de 